\title{
The influence of longitudinal elastic systems on the celerity (speed of elastic waves)
}

\author{
I. Omer, D. I. Arsenie \& M. Florea \\ Civil Engineering Faculty, Ovidius University Constanta, Romania
}

\begin{abstract}
This paper presents two elastic systems which protect the pipe by reducing the celerity. We propose two options for protection: the first solution named "barrel jacket", where the wall pipe consists of an elastic inner layer soldered in the steel pipe (rubber) which has no role in resistance and the second solution named "elastic cylinder", where in the ordinary pipe, is disposed longitudinally a cilinder with small section, fabricated from an elastic material (rubber). The common idea is that the overpressure strain the elastic element and so the flow area increase while the celerity decrease. We give the formula for celerity in the both cases and two graphical representation which showes the dependence of the celerity on the given parameters.
\end{abstract}

Keywords: celerity (speed of elastic waves), pipe, overpressure, elastic element, rapid closure.

\section{Introduction}

The protection of hydraulic systems to the dangerous effects of the water hammer can be assured by many solutions.

The more frequent solutions for protection used in Romania, strictly from technical point of view, are the surge tank and the air chamber.

The surge tank is a solution which is applied specially in the hydro energetically domain and the air chamber is usually used for the protection of pumping installation.

The both solutions are expensive, the air chamber having furthermore the disadvantage of a pretentious exploitation because it is necessary to maintain the water level and the pressure in the air chamber at the optimal values. 
The paper examines two variants, unusual in Romanian hydrotechnics practice, for protection against water hammer (hydraulic shock) caused by rapid closure valves.

Both protection solutions are based on reducing the speed of propagation of elastic waves (celerity) in their transition through a part of the pipeline with special properties. Under the action of overpressures this elastic element is compressed increasing the flow section much more than in a steel pipe.

Assumptions that taken into account are:

- tension in the pipe wall is flat;

- the material is elastic;

- it only considers efforts stretching from pipe walls, calculated effort to make the boiler is considered that the walls are thin pipeline, efforts nepreluând flexural.

To achieve this goal in this paper are considered the following two solutions:

a) "barrel jacket"

the wall pipe consists of an elastic inner layer soldered in the steel pipe (rubber) which has no role in resistance.

b) "elastic cylinder"

In the ordinary pipe, is disposed longitudinally a cilinder with small section, fabricated from an elastic material (rubber).

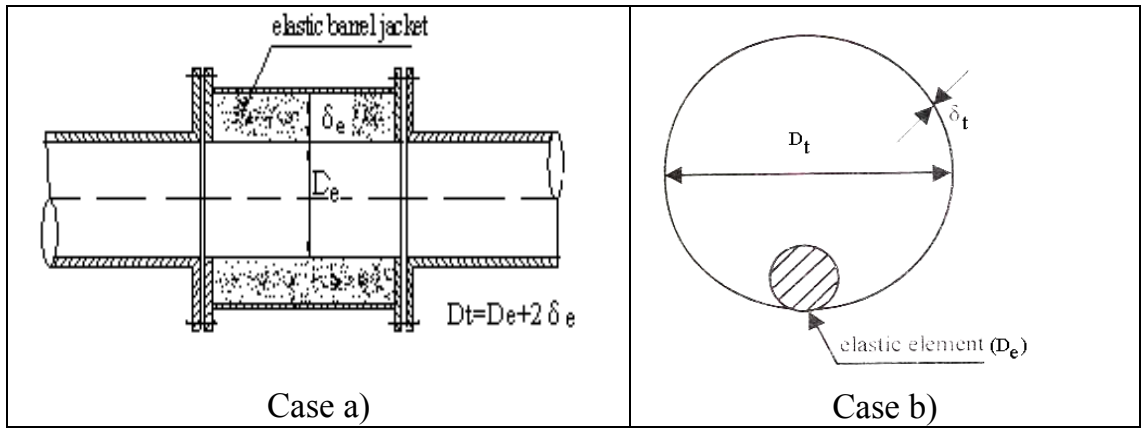

Figure 1: Cross section through the pipe.

\section{Mathematical model}

To obtain a celerity formula, we start with expression in two ways of the variation of fluid mass in the time interval $\Delta t$, mass which is between two control sections, at distance $l=c \Delta t, c$ - celerity, $\Delta t$ - interval time.

$$
m=\rho A \Delta l \Rightarrow \Delta m=\Delta(\rho A) \Delta l \Delta t
$$

where the A is the flow section, $\rho$ - the water density.

If we consider the case of total instant closing of the valve, it will result:

$$
\Delta m=\rho A v_{0} \Delta t
$$

where $v_{0}$ - initial mean speed of the water. 
From equalizations of the two expressions results first relation of celerity:

$$
c=\frac{v_{0}}{\frac{\Delta \rho}{\rho}+\frac{\Delta A}{A}}
$$

Furthermore the mathematical model for the two solutions is different because the $\frac{\Delta A}{A}$ ratio is calculated in another way.

\subsection{Solution a) - "barrel jacket"}

The overpressure $(\Delta p)$ produces a variation of the elastic element thickness $\Delta \delta_{e}$ and a diameter variation of pipe wall $\Delta D_{t}$ so the relative variation flow section becomes:

$$
\frac{\Delta A}{A}=\frac{4\left(\Delta D_{t}^{2}+\Delta \delta_{e}^{2}+D_{e} \frac{\Delta D_{t}}{2}+D_{e} \Delta \delta_{e}+\Delta D_{t} \Delta \delta_{e}\right)}{D_{e}^{2}}
$$

The compression strain of the elastic barrel jacket is:

$$
\Delta \delta_{e}=\frac{\Delta p \delta_{e}}{E_{e}}
$$

where $E_{e}$ - elasticity coefficient of the elastic element.

The elongation of the pipe's diameter is:

$$
\Delta D_{t}=\frac{\Delta p D_{t}^{2}}{2 \delta_{t} E_{t}}
$$

where $E_{t}$ - elasticity coefficient of steel.

The relative variation of water density is:

$$
\frac{\Delta \rho}{\rho}=\frac{\Delta p}{E_{w}}
$$

where $E_{w}$ - elasticity coefficient of water.

After algebra calculus we obtain the final formula:

$$
c=\frac{\sqrt{\frac{E_{w}}{\rho}}}{\sqrt{1+\frac{D_{t}^{2} E_{w}}{\delta_{t} D_{e} E_{t}}+\frac{4 \delta_{e} E_{w}}{D_{e} E_{e}}+\frac{\Delta p}{D_{e}}\left(\frac{D_{t}^{4} E_{w}}{\delta_{t}^{2} D_{e} E_{t}^{2}}+\frac{4 \delta_{e}^{2} E_{w}}{D_{e} E_{e}^{2}}+\frac{2 \delta_{e} E_{w} D_{t}^{2}}{D_{e} E_{e} E_{t} \delta_{t}}\right)}}
$$

Numerical calculus with usual values of physical parameters from this formula indicates that several terms are relative very small and may be neglected. So we obtain the approaching implicit formula becomes: 


$$
c=\frac{1400}{\sqrt{1+\frac{4 \delta_{e} E_{w}}{D_{e} E_{e}}+c \frac{4 \rho v_{0} \delta_{e}^{2} E_{w}}{D_{e}^{2} E_{e}^{2}}}}
$$

Also we found that the variation of initial speed $\left(\mathrm{v}_{0}\right)$ has a small influence on the celerity than we made the graphical representation (figure 2) for constant value $v_{0}=2 \frac{\mathrm{m}}{\mathrm{s}}$.

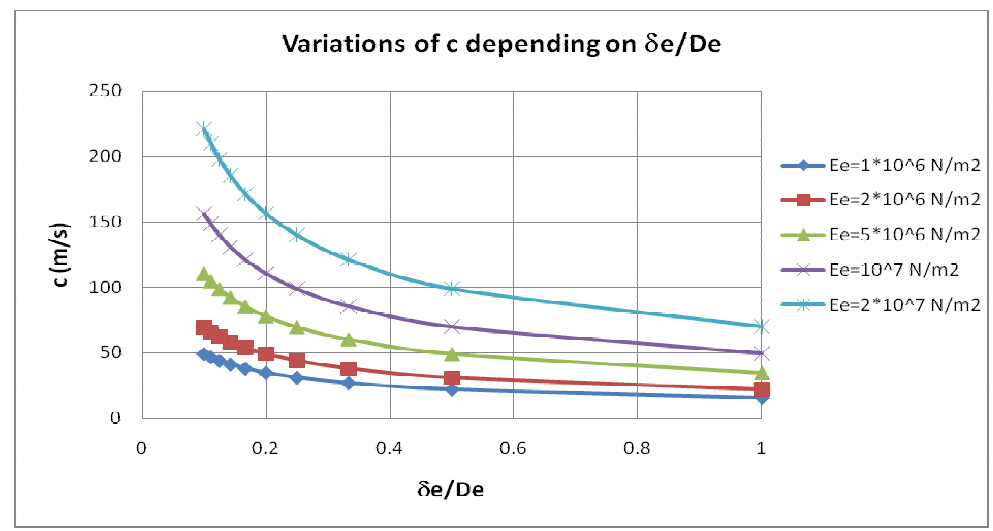

Figure 2: Variations of $\mathrm{c}$ depending on $\delta \mathrm{e} / \mathrm{De}$ for different values of $\mathrm{E}_{\mathrm{e}}$

\subsection{Solution b) - "elastic cylinder"}

In this case, the relative variation of the elastic element section is:

$$
\frac{\Delta A_{e}}{A_{e}}=\frac{\Delta p}{E_{e}}
$$

and the relative variation of the circular section of the pipe is:

$$
\frac{\Delta A_{c}}{A_{c}}=\frac{\Delta p D_{t}}{E_{c} \delta_{t}}
$$

Adding the absolute values of the section variations we obtain:

$$
\Delta A_{t}=\frac{\pi \Delta p}{4}\left(\frac{D_{t}^{3}}{E_{c} \delta_{t}}+\frac{D_{e}^{2}}{E_{e}}\right)
$$

The relative variation of the flow section is:

$$
\frac{\Delta A_{t}}{A_{t}}=\frac{\Delta p\left(\frac{D_{t}^{3}}{E_{c} \delta_{t}}+\frac{D_{e}^{2}}{E_{e}}\right)}{D_{t}^{2}-D_{e}^{2}}
$$


After algebra calculus we obtain the final formula:

$$
c=\frac{\sqrt{\frac{E_{w}}{\rho}}}{\sqrt{1+\frac{E_{w} D_{t}}{E_{c} \delta_{t}}\left(D_{t}^{2}+\frac{E_{c}}{E_{e}} \frac{D_{e}^{2} \delta_{t}}{D_{t}}\right)}}
$$

Comparing this formula with the formula of celerity for a steel pipe $c=\frac{\sqrt{\frac{E_{w}}{\rho}}}{\sqrt{1+\frac{E_{w} D_{t}}{E_{c} \delta_{t}}}}$, we introduce the notation $k=\frac{D_{t}^{2}+\frac{E_{c}}{E_{e}} \frac{D_{e}^{2} \delta_{t}}{D_{t}}}{D_{t}^{2}-D_{e}^{2}}$, the relation (14) takes the following form:

$$
c=\frac{1400}{\sqrt{1+k \frac{E_{w} D_{t}}{E_{c} \delta_{t}}}}
$$

Using this formula we made the graphical representation (figure 3) which gives the celerity as a function of De/Dt and $\mathrm{E}_{\mathrm{e}}$.

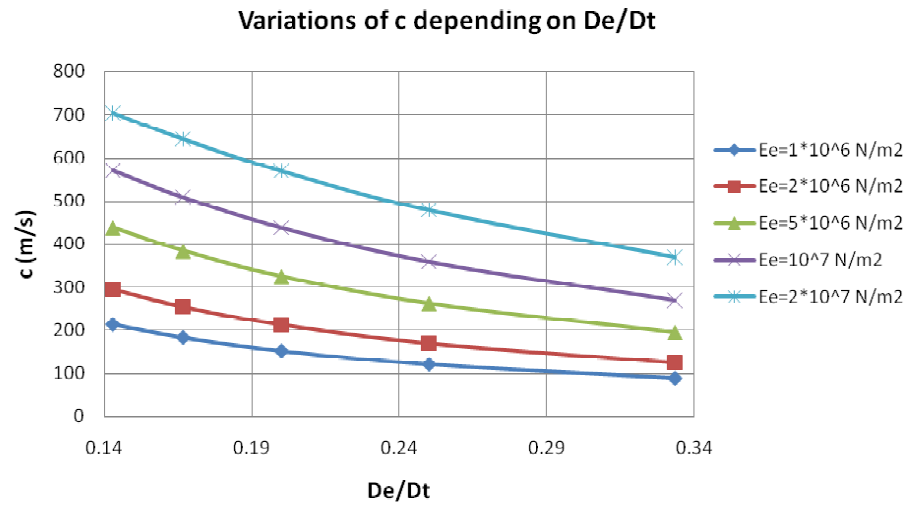

Figure 3: Variations of $\mathrm{c}$ depending on De/Dt for different values of $\mathrm{E}_{\mathrm{e}}$.

\section{Numerical test}

We consider the following example test:

a) First solution: $\delta_{e}-$ variable between $15 \mathrm{~mm}$ and $300 \mathrm{~mm}$, $D_{e}=600 \mathrm{~mm}, E_{e}=5 \cdot 10^{6} \mathrm{~Pa}, E_{w}=2 \cdot 10^{9} \mathrm{~Pa}, \rho_{w}=10^{3} \mathrm{~kg} / \mathrm{m}^{3}$, $v_{0}=2 \mathrm{~m} / \mathrm{s}$; 
b) Second solution: $D_{e}-$ variable between $15 \mathrm{~mm}$ and $300 \mathrm{~mm}$, $D_{t}=600 \mathrm{~mm}, E_{e}=5 \cdot 10^{6} \mathrm{~Pa}, E_{w}=2 \cdot 10^{9} \mathrm{~Pa}, E_{c}=2 \cdot 10^{11} \mathrm{~Pa}$, $\rho_{w}=10^{3} \mathrm{~kg} / \mathrm{m}^{3}, \delta_{t}=7 \mathrm{~mm} ;$

The results are presented in figure 4 .

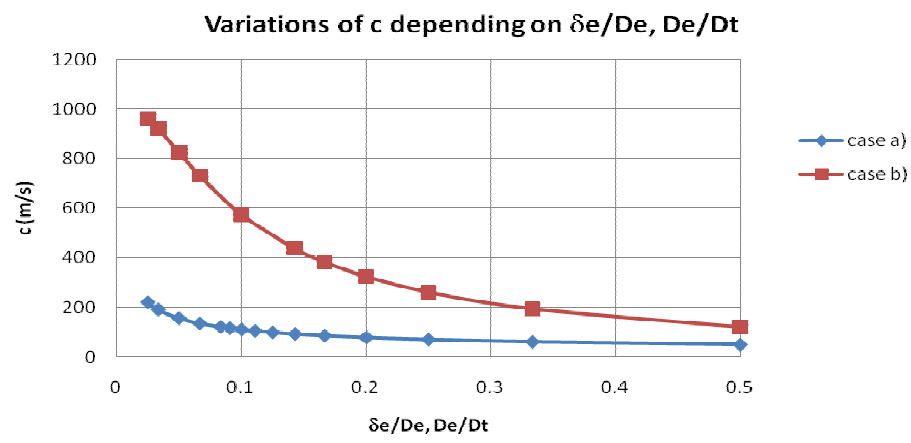

Figure 4: Variation of the celerity for the example test.

\section{Conclusions}

We see from the graphical representations (figure 2 and figure 3) that the elasticity coefficient of the elastic element is an important parameter; by increasing this elasticity coefficient involves the increasing of the celerity.

By increasing the volume of the elastic material, respective by increasing $\delta$ e (case a) or De (case b) the celerity decreases. The variation of the celerity is bigger in case $b$ than in case $a$.

The values of the celerity are small in the case a comparing by the case $b$.

Taking account that in case $b$ the elastic cylinder reduces the flow section in stationary state, we appreciate that the case a "barrel jacket" is more efficient than case $b$.

These theoretical results must be validated by experimental researches.

The present paper is realized in the framework of one contract cod 1219 , financing by "Romanian Executive Unity for Financing Higher Education and Scientific Research".

\section{References}

[1] Popescu, M., Arsenie, D.I. \& Vlase, P., Applied Hydraulic Transients - For Hydropower Plants and Pumping Stations, Balkema, Netherlands, 2003. 
[2] Arsenie, D.I., Florea, M., Mîrzali, I., Nițescu, C., "Some aspects concerning the propagation of the water hammer in pressure pipes", Timişoara, Romania, 2005.

[3] Arsenie, D.I., Florea, M., Omer, I., "Hydraulic system protection from the water hammer using a longitudinal elastic element", Cluj, pp. 303-308, Romania, 2003.

[4] Chaudry, H., "Applied hydraulic transients", Van Nastrand Reinhold Company, 1987.

[5] Streeter, V., Wylie, E., "Hydraulic transients", Mc. Graw Hill, New York, 1967. 